\title{
Modeling Combined Heat Transfer in the Operating Area of the High Power Heating Test Facility
}

\author{
Riza Baldzhiev $^{1, *}$, Pavel Prosuntsov ${ }^{1}$ \\ ${ }^{1}$ Bauman Moscow State Technical University, 105005 Moscow, Russia
}

\begin{abstract}
The operating temperatures for the current aerospace heat shield materials exceed $2000 \mathrm{~K}$ [1], which makes their experimental testing a complex and expensive issue requiring specialized testing facilities capable to obtain the temperature level in question. In most cases, the thermal tests of the materials samples and thermal shield elements are conducted on the radiative heating test facilities. Generally, these facilities use tungsten halogen lamps (THL) [2, 3]. However, maximal operating temperatures for such type facilities do not exceed $1500 \mathrm{~K}$ due to the tungsten-halogen cycle disruption. The main challenge is to comply with the bulbs thermal conditions, which must remain below $1270 \mathrm{~K}$ [1]. This paper outlines the layout of the working area of the THL radiative heating facility, where the test specimen temperature is increased to $2100 \mathrm{~K}$ due to the compressed air flow over the bulbs, which for a considerable part solves the problem of the thermal behavior simulation.
\end{abstract}

\section{Modeling combined heat transfer}

The facility design layout required solving the complex problem of simulating the thermal processes in the operating area, which must take into account for the three interrelated heat transfer processes: heat conductivity in the test specimen, heat insulation, heaters, bulbs and glass partitions; convection in the flowing gas medium and radiation in the semi-transparent bodies and the gas medium. Apart from this, the spectral and temperature functions of the thermal physical and optical characteristics were taken into account.

Since the longitudinal dimension $(250-400 \mathrm{~mm})$ of the heating sources exceeds their lateral dimension $(11 \mathrm{~mm})$ by an order of magnitude, the heat transfer problem can be solved two-dimensionally, which will mean a significant decrease in the required processing capacity. For the radiative heat transfer simulation we used the discreteordinates method with 16 angular dimensions, which enabled accurately accounting for the radiative heat transfer in the quartz glasses [4] and THL casings. It was assumed that the gas flow is turbulent in nature and is described by K-eps model. The modelling was conducted in the finite-element package ANSYS Fluent [5] (license 339001). The characteristic dimension of the elements was about $1 \mathrm{~mm}$, and the total amount of elements

*Corresponding author: lexiusb@gmail.com 
- about 30000 pcs. The time increment was $0.01 \mathrm{~s}$, and the total process duration was $600 \mathrm{~s}$, the duration of computations varied from 20 to 30 hours on advanced computers (Intel Core i7-4790 processor).

The operating area of the high-power heating test facility (Fig. 1) comprises the following components: heat-insulating blocks $(1,7)$, radiative screen from the polished heat-resistant steel (2), nine HTLs, each $2.0 \mathrm{~kW}$ in capacity, a system of glass partitions (4, 5), the test specimen (6).

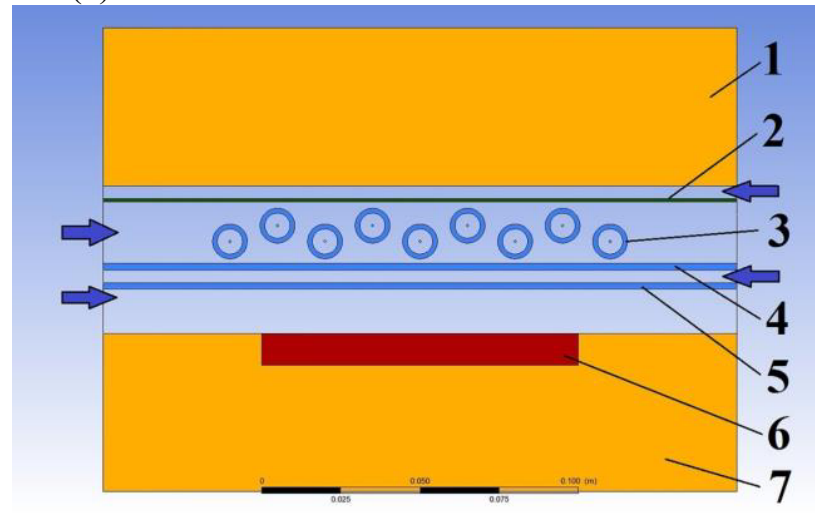

Fig. 1. Geometric model of the test facility working area: 1,7) upper and lower heat insulation blocks; 2) radiative screen: 3) THL; 4, 5) upper and lower glass partitions; 6) test specimen.

The working area was divided into several zones, so that the flow and the direction of the gas fluxes can be varied (the fluxes directions are indicated with arrows in Fig. 1). The choice of the fluxes directions and the symmetrical positioning of the heaters (relative to the test specimen) helps to achieve the sufficient uniformity of the temperature field over the specimen surface. The effective heat transfer away from the bulbs was a result of the THL checkerboard positioning at a relatively small distance to the partitions and screen result.

The modelling results (Fig. 2,3) demonstrate that the specimen temperature reaches $2100 \mathrm{~K}$, with $4 \%$ non-uniformity of the temperature distribution. The temperature of the THL bulbs as well as the temperature of other components do not exceed the permitted values $(1200 \mathrm{~K}$ for the THL bulbs and glass partitions, $1300 \mathrm{~K}$ for the heat-resistant steel, $1500 \mathrm{~K}$ for the heat insulation blocks). Maximum values of the gas flux velocity are found in the clearances between the THL bulbs and the glass partitions and (or) the radiative screen, constituting $20 \mathrm{~m} / \mathrm{s}$, the mass-average velocity is around $7 \mathrm{~m} / \mathrm{s}$. The total gas flow on the facility elements cooling is about $0.05 \mathrm{~kg} / \mathrm{s}$.

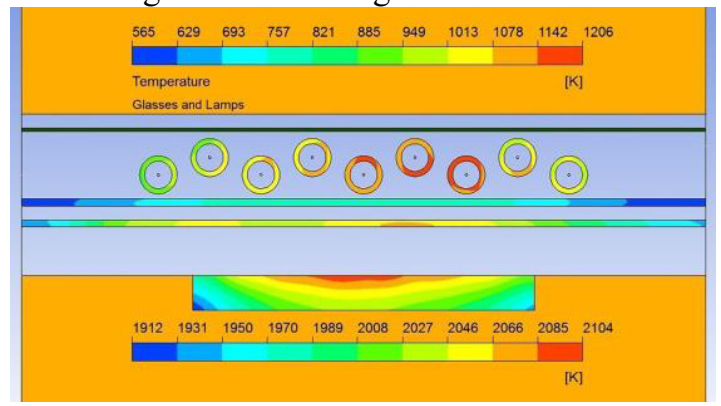

Fig. 2. Temperature fields in the bulbs, glass partitions and the specimen, K. 


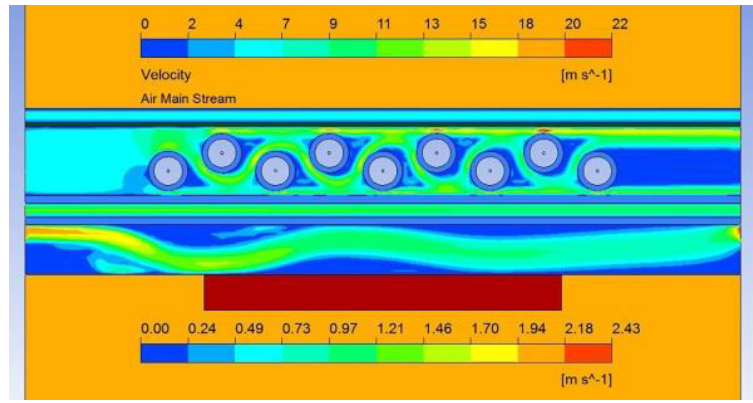

Fig. 3. The gas velocity in the model, $\mathrm{m} / \mathrm{s}$.

One of the advantages of the proposed layout is the possibility to simulate the highpower cooling of the specimen by supplying gas into the zone above it. Fig. 4 shows the temperature change in specimen surface centre as a function of time. The maximum heating rate was $140 \mathrm{~K} / \mathrm{s}$, and the maximum cooling rate was $110 \mathrm{~K} / \mathrm{s}$. It was considered that the heating was conducted in 300 seconds, after which the THLs were switched off and the gas was supplied into the zone above the test specimens at $20 \mathrm{~m} / \mathrm{s}$.

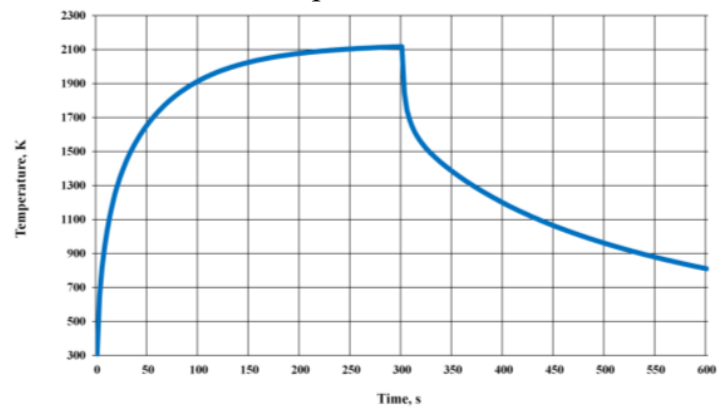

Fig. 4. Temperature on the specimen surface as a function of time.

\section{Modification of the operating area}

The temperatures distribution over the specimen surface can be more uniform if the amount of THL is increased up to 13 pcs (Fig. 5). The heaters are then divided into four groups: three groups of 3 lamps and one group of 4 lamps. The output of the leftmost group is specified for this type THL constituting $2.0 \mathrm{~kW}$, the output of the next two groups is $50 \%$ and $60 \%$ of the nominal, while the rightmost is $75 \%$ of the nominal output. The other parameters were remained the same. Thus, the non-uniformity of the surface temperature distribution can be reduced to $2.5 \%$. The modelling results are presented at Fig. 6 .

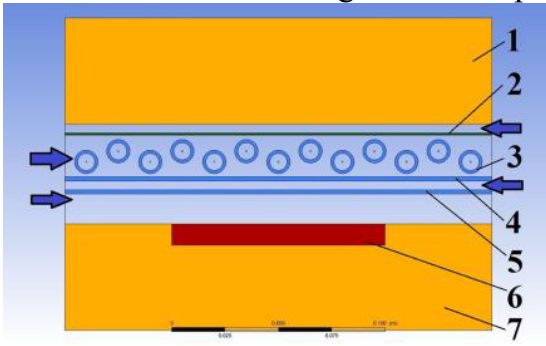

Fig. 5. Geometric model of the test facility working area with added THL: 1,7) upper and lower heat insulation blocks; 2) radiative screen: 3) THL; 4, 5) upper and lower glass partitions; 6) test specimen. 


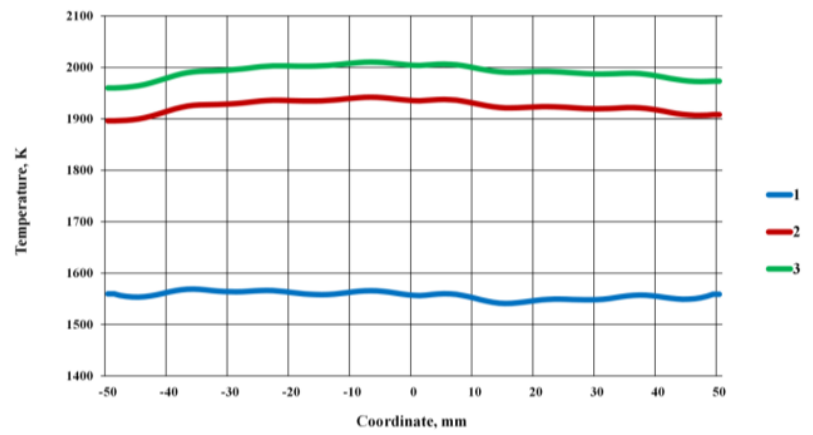

Fig. 6. Surface temperature distribution at various points in time, K: 1) $60 \mathrm{~s}$; 2) $180 \mathrm{~s}$; 3) $300 \mathrm{~s}$.

\section{Pneumatic system}

To enable the reliable operation of described test facility, the gas should be supplied at a steady rate with the specified velocity, with the hot gas effectively removed from the working area. This challenge required designing a pneumatic system (Fig. 7) which comprises a gas supply duct decreasing the non-uniformity of the velocity fields at the input to no more than $5 \%$, and a hot gas disposal duct decreasing the mass-average temperature at the facility exit to $365 \mathrm{~K}$. The temperature behavior of the system elements was simulated and the requirements to the pneumatic system materials were specified.

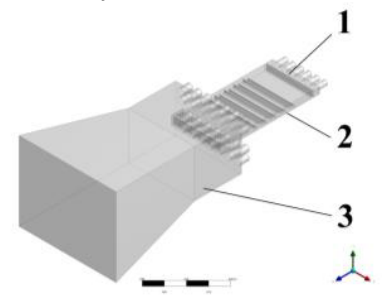

Fig. 7. Pneumatic system: 1) gas supply duct; 2) working area; 3) hot gas disposal tract.

\section{Conclusion}

Thus, it was verified theoretically that the power of the THL heating facilities could be increased by means of the dynamic air flow. The operable model of a perspective facility was proposed for testing heat shield materials with operating temperatures up to $2100 \mathrm{~K}$.

\section{References}

1. V.N. Yeliseev, V.A. Tovstonog, Vestnik MGTU im. Baumana. Ser. Mashinostroenie, 1, 57 (2011) [In Russian]

2. Yu.V. Polezhaev, S.V. Reznik, A.N. Baranov, Materials and coatings under extreme condition (Bauman MSTU Publ., Moscow, 2002)

3. A.N. Baranov, A.G. Belozerov, Yu.S. Il'in, V.F. Kut'inov, Static strength test of supersonic aircraft (Mashinostroenie, Moscow, 1974) [In Russian]

4. P.V. Prosuntsov, I.A. Mayorova, A.V. Zuev, Thermal Processes in Engineering, 6, (2) 317 (2014)

5. ANSYS Fluent [Electronic resource] / Ansys. - 2017 - Access mode: http://www.ansys.com/Products/Fluids/ANSYS-Fluent. 